\title{
Anodic titanium oxide: A new template for the synthesis of larger diameter multi-walled carbon nanotubes
}

\author{
I. Eswaramoorthi, Lian-Pin Hwang * \\ Institute of Atomic and Molecular Sciences, Academia Sinica and Department of Chemistry, National Taiwan University, Taipei 106, Taiwan
}

Received 16 June 2006; received in revised form 20 December 2006; accepted 22 December 2006

Available online 4 January 2007

\begin{abstract}
Carbon nanotubes (CNTs) with larger diameter were synthesized over anodic titanium oxide (ATO) template by CVD method using acetylene as carbon source. The porous titanium oxide was obtained by anodization of titanium metal in a mixture of $1 \mathrm{M} \mathrm{H}_{2} \mathrm{SO}_{4}+0.5 \% \mathrm{HF}$ electrolyte at a constant applied potential of $40 \mathrm{~V}$. The XRD analysis of anodized titanium revealed that rutile and anatase forms of $\mathrm{TiO}_{2}$ are formed due to anodization. Further, SEM analysis was used to follow the development of pores on titanium surface. The TEM analysis revealed that the formed CNTs are straight and hollow with uniform wall thickness as well as larger diameter $(70-80 \mathrm{~nm})$. HRTEM study showed that the formed CNTs are multi-walled and their wall thickness is around $2-3 \mathrm{~nm}$. Further, the structural features of the formed CNTs were studied by XRD. Raman spectroscopy was used to study the degree of graphitization of CNTs. The Lewis acid sites of $\mathrm{TiO}_{2}$ present in the internal surface of the pores play an important role in the catalytic decomposition of acetylene and hence the formation of CNTs. When increasing the carbon deposition time, the wall thickness of CNTs is not increased significantly, indicating that the decomposition of acetylene is due to Lewis acid sites of TiO ${ }_{2}$ and not due to thermal decomposition. Further, the morphology of CNTs formed over ATO template was compared with that of CNTs formed on Co electrodeposited ATO. There is no significant difference in morphology as well as wall thickness was observed between the CNTs grown over ATO with and without Co catalyst. But, still further investigations are necessary to study the structural differences between the CNTs grown over ATO with and without Co catalyst.
\end{abstract}

(C) 2007 Elsevier B.V. All rights reserved.

Keywords: Nanotubes; Chemical vapor deposition; Morphology; Nanotechnology

\section{Introduction}

Carbon nanotubes (CNTs) are tubular $\mathrm{sp}^{2}$-like carbons with nanoscale dimensions that exhibit fascinating electrical, mechanical, thermal and optical properties which make them very promising in various potential applications [1-9]. Extensive efforts have been made to control the growth and properties of CNTs. Different growth techniques including arc discharge [10], plasma techniques [11,12], laser ablation [13] and pyrolysis of hydrocarbon vapor over various catalysts [14] have been used to control the position, direction and arrays of CNTs. Recently, growth of CNTs over porous template such as anodic aluminum oxide (AAO) inspired a new way to grow well

\footnotetext{
* Corresponding author. Tel.: +886 23366 1166; fax: +886 233663294 .

E-mail address: nmra@po.iams.sinica.edu.tw (L.-P. Hwang).
}

ordered CNTs with uniform size and shape due to the well ordered pore structure with narrow size distribution and uniform inter-pore spacing $[15,16]$. The AAO template with various pore size and distribution patterns can be easily obtained by varying the oxidation voltage and the acid electrolyte and hence electrochemically prepared porous alumina is considered as one of the most important template material. Highly ordered graphite nanofibers were formed by pyrolysis of ethylene followed by annealing at a temperature less than $600^{\circ} \mathrm{C}$ over Nicatalyst activated AAO [17]. Ultrafine carbon tubes were formed in AAO without catalyst by pyrolytic carbon deposition from propylene at $800^{\circ} \mathrm{C}[18]$. The obtained CNTs showed low crystallinity, but with certain atomic plane orientation in the carbon tubes. Electrochemically deposited cobalt particles in the pore bottom of the AAO template resulted in the formation of CNTs by acetylene pyrolyzing at $650{ }^{\circ} \mathrm{C}$ [15]. The proposed mechanisms were that cobalt particles might promote the 
decomposition or initiate the tube growth through the initial catalytic decomposition [19]. Naturally, the question arises whether other value metals such as $\mathrm{Ti}, \mathrm{Ta}, \mathrm{Nb}, \mathrm{V}, \mathrm{Hf}$ and $\mathrm{W}$ can also be used to grow the CNTs inside the pore arrays fabricated by the anodization process, similar to AAO. The surface of these value metals is usually covered with a native oxide film of a few nanometers when these metals are exposed to oxygen containing surrounding. Current state-of-the-art techniques for aluminum anodization using self-assembly of pores allow the fabrication of monodomain and monodisperse porous alumina structures with adjustable pore size and depth. Generally, the formation of highly ordered pores is based on lithographic definition of etch initiation sites or be completely due to selforganization. However, porous templates based on other value metals by the anodization process have been rarely studied. Among the value metal oxides, titanium dioxide is considered as a promising material for photocatalysis, catalyst support and implants [20,21]. Zwilling et al. [22,23] reported that a porous $\mathrm{TiO}_{2}$ layer is created by anodization in a chromic acid solution in the presence of a small amount of HF, whereas only the barrier $\mathrm{TiO}_{2}$ layer is formed in pure chromic acid contrary to porous alumina. The compact films grown in chromic acid solution are amorphous and the porous films grown in the chromic acid-HF solution are partially crystalline. The growth mechanism in fluorinated electrolyte involves a competition between dissolution and oxide formation, in which the $\mathrm{Cr}^{\mathrm{VI}}$ ions and fluorine species play locally a respective poisoning and antidote role, resulting in the growth of porous film. Further they reported that the thickness of $\mathrm{TiO}_{2}$ barrier oxide is independent of anodization time, whereas that of the porous oxide of titanium increases with anodizing time as well as applied voltage. Gong et al. [24] studied the anodic oxidation of $\mathrm{Ti}$ in aqueous solution containing 0.5 to $3.5 \mathrm{wt} \% \mathrm{HF}$. The obtained tubes are well aligned and organized into high-density uniform arrays with barrier layer at their bottom. The tube diameter $(25-65 \mathrm{~nm})$ was found to increase with anodizing voltage. Further they claimed that the final thickness (or length of $\mathrm{TiO}_{2}$ tubes) of porous $\mathrm{TiO}_{2}$ structure formed in diluted $\mathrm{HF}$ solution is independent of the anodizing time. Recently, Beranek et al. [25] produced highly ordered porous $\mathrm{TiO}_{2}$ in $\mathrm{H}_{2} \mathrm{SO}_{4}$ electrolyte containing 0.15 wt.\% HF. However, the thickness of the pores is still limited up to $500 \mathrm{~nm}$. Also, Choi et al. [26] compared the anodization of nanoimprinted titanium with porous alumina and found that the anodized titanium films have more complicated morphologies than anodized alumina. Due to the semiconducting properties of $\mathrm{TiO}_{2}$, a mirror image of the behavior of porous alumina can not be accomplished. Since, $\mathrm{TiO}_{2}$ is widely used as catalyst, photocatalyst and catalyst support in a number of chemical transformations, the porous $\mathrm{TiO}_{2}$ obtained by the anodization process can also be used as a template as well as a catalytic material for the growth of CNTs. The possibility to prepare ordered porous $\mathrm{TiO}_{2}$ by anodization and their Lewis acid sites are considered as important features to grow CNTs. Up to now no report is available on the usage of anodized $\mathrm{TiO}_{2}$ as template for CNTs growth. Hence, in the present study, we aimed to grow the CNTs over anodized $\mathrm{TiO}_{2}$ by CVD method and to study their morphology and structural features using various instrumental techniques and to compare the morphology of CNTs grown with and without Co catalyst over porous $\mathrm{TiO}_{2}$ template.

\section{Experimental}

\subsection{Anodization of titanium}

A piece of commercially pure titanium metal (Giedmann, $99.5 \%$ purity) was washed with acetone and distilled water and dried in $\mathrm{N}_{2}$ atmosphere. The native oxide already formed on $\mathrm{Ti}$ metal surface was removed by chemical treatment with a mixture of $\mathrm{HNO}_{3} / \mathrm{HF} / \mathrm{H}_{2} \mathrm{O}$ in a ratio of $3: 1: 6$ at $60-70{ }^{\circ} \mathrm{C}$ for $30 \mathrm{~min}$ and washed with distilled water and dried in $\mathrm{N}_{2}$ atmosphere. The chemical polishing was chosen as the electrolytic polishing proved to lead to non-homogeneous films [22]. The anodization cell is a two-electrode system consisting of a graphite counter electrode and the titanium metal piece as working electrode, electrolyte of $1 \mathrm{M} \mathrm{H}_{2} \mathrm{SO}_{4}$ with 0 , 0.25 and $0.5 \%$ HF solution contained in a glass chamber and an extended range DC power supply system. The chemically polished titanium metal piece was mounted as anode and graphite serves as cathode. Then they were immersed in the glass chamber containing electrolyte maintained at $1{ }^{\circ} \mathrm{C}$ and subjected to anodization by applying constant potential of $40 \mathrm{~V}$ for a desired time. Care was taken to maintain constant temperature and velocity of stirring of electrolyte during the anodization. After the completion of anodization, the titanium metal piece was taken out and washed with distilled water and dried in $\mathrm{N}_{2}$ atmosphere. The electrodeposition of cobalt over the obtained anodic titanium oxide (ATO) template was carried out with an aqueous solution of $\mathrm{CoSO}_{4} \cdot 7 \mathrm{H}_{2} \mathrm{O}(240 \mathrm{~g} / \mathrm{l})$ and boric acid (40 g/l) for comparison purposes.

\subsection{Characterisation of ATO}

The anodized titanium oxide was analysed by X-ray diffraction on X'Pert PRO-XRD X-ray diffractometer using $\mathrm{CuK} \alpha$ radiation $(\lambda=0.1541 \mathrm{~nm})$ in the range of $10-60^{\circ}$. Also, the surface morphology of the porous titanium film was analysed by field-enhanced scanning electron microscopy (FESEM) in LEO 1530 FE-SEM instrument.

\subsection{Growth and characterisation of CNTs}

The CNTs were grown over an ATO template by CVD method. The ATO template is taken in a ceramic boat and kept in a quartz tube and heated to $650{ }^{\circ} \mathrm{C}$ in $\mathrm{Ar}$ atmosphere. The carbon source acetylene gas $(30 \mathrm{sccm})$ is mixed with $\mathrm{Ar}$ $(100 \mathrm{sccm})$ and passed into the quartz tube at $650{ }^{\circ} \mathrm{C}$ for $2 \mathrm{~h}$. Then the acetylene gas is turned off and $\mathrm{Ar}$ gas flow is continued for another $15 \mathrm{~h}$ at $600{ }^{\circ} \mathrm{C}$ (annealing). After $15 \mathrm{~h}$, the system is cooled to room temperature with Ar flow. Then the sample is taken out from the boat and treated with $23 \% \mathrm{HF}$ for $30 \mathrm{~h}$, filtered and washed in order to remove the ATO template. The morphology of the CNTs grown over the ATO template was analysed by SEM analysis. The CNTs grown over the ATO 


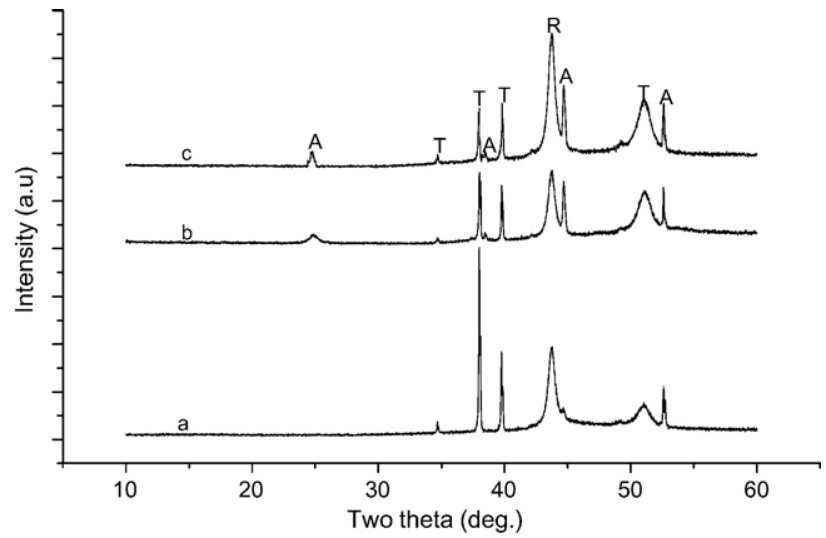

Fig. 1. XRD patterns of titanium oxide anodized in different conditions; (a) in $1 \mathrm{M} \mathrm{H}_{2} \mathrm{SO}_{4}$; (b) in $1 \mathrm{M} \mathrm{H}_{2} \mathrm{SO}_{4}+0.25 \% \mathrm{HF}$; (c) in $1 \mathrm{M} \mathrm{H}_{2} \mathrm{SO}_{4}+0.5 \% \mathrm{HF}$ for $72 \mathrm{~h}$ at $40 \mathrm{~V}$ (T-Titanium, A-Anatase, $\mathrm{R}$-Rutile).

template, as they were, were cleaned with ethanol and subjected to SEM analysis in Hitachi SEM instrument operating at $50 \mathrm{kV}$. The features of the CNTs were studied by transmission electron microscope (TEM) using Hitachi H-800 instrument at an accelerating voltage of $150 \mathrm{kV}$. In order to get more insights about the wall structure of CNTs, high-resolution TEM (HRTEM) images were also obtained on Philips TECNAI F30 FEG instrument at an accelerating voltage of $300 \mathrm{kV}$. For both TEM and HRTEM, the CNT sample was dispersed in dry ethanol with an ultra-sonic machine and then deposited on a copper grid with a holey carbon film. Using X'Pert PRO-XRD instrument with $\mathrm{CuK} \alpha$ radiation $(\lambda=0.1541 \mathrm{~nm})$, the XRD pattern of the purified CNT sample was recorded in the range $20-50^{\circ}$ with a scan rate of $0.050640^{\circ} / \mathrm{s}$. Further, the vibrational characteristics of CNTs were recorded with Raman spectroscopy (Renishaw) in the range $1000-2000 \mathrm{~cm}^{-1}$.

\section{Results and discussion}

\subsection{XRD and SEM analyses of anodized titanium oxide}

The anodization of $\mathrm{Ti}$ was carried out with varying $\mathrm{HF}$ concentrations in order to get well ordered porous $\mathrm{TiO}_{2}$. The XRD patterns of the anodized $\mathrm{TiO}_{2}$ in $1 \mathrm{M} \mathrm{H}_{2} \mathrm{SO}_{4}$ with different amounts $(0,0.25$ and $0.5 \%)$ of $\mathrm{HF}$ at $40 \mathrm{~V}$ for $72 \mathrm{~h}$ are presented in Fig. 1. It claims the presence of both anatase and rutile phases of $\mathrm{TiO}_{2}[27,28]$. It is observed that peaks corresponding to $\mathrm{Ti}$ are dominating than peaks for titanium oxide (Fig. 1a), indicating that anodization in $1 \mathrm{M} \mathrm{H}_{2} \mathrm{SO}_{4}$ does not lead to the formation of more titanium oxide. When $0.25 \% \mathrm{HF}$ is introduced in the electrolyte system, the intensity of the peaks (Fig. 1b) corresponding to the anatase and rutile phase increases significantly. Further, additional peaks for anatase phase are

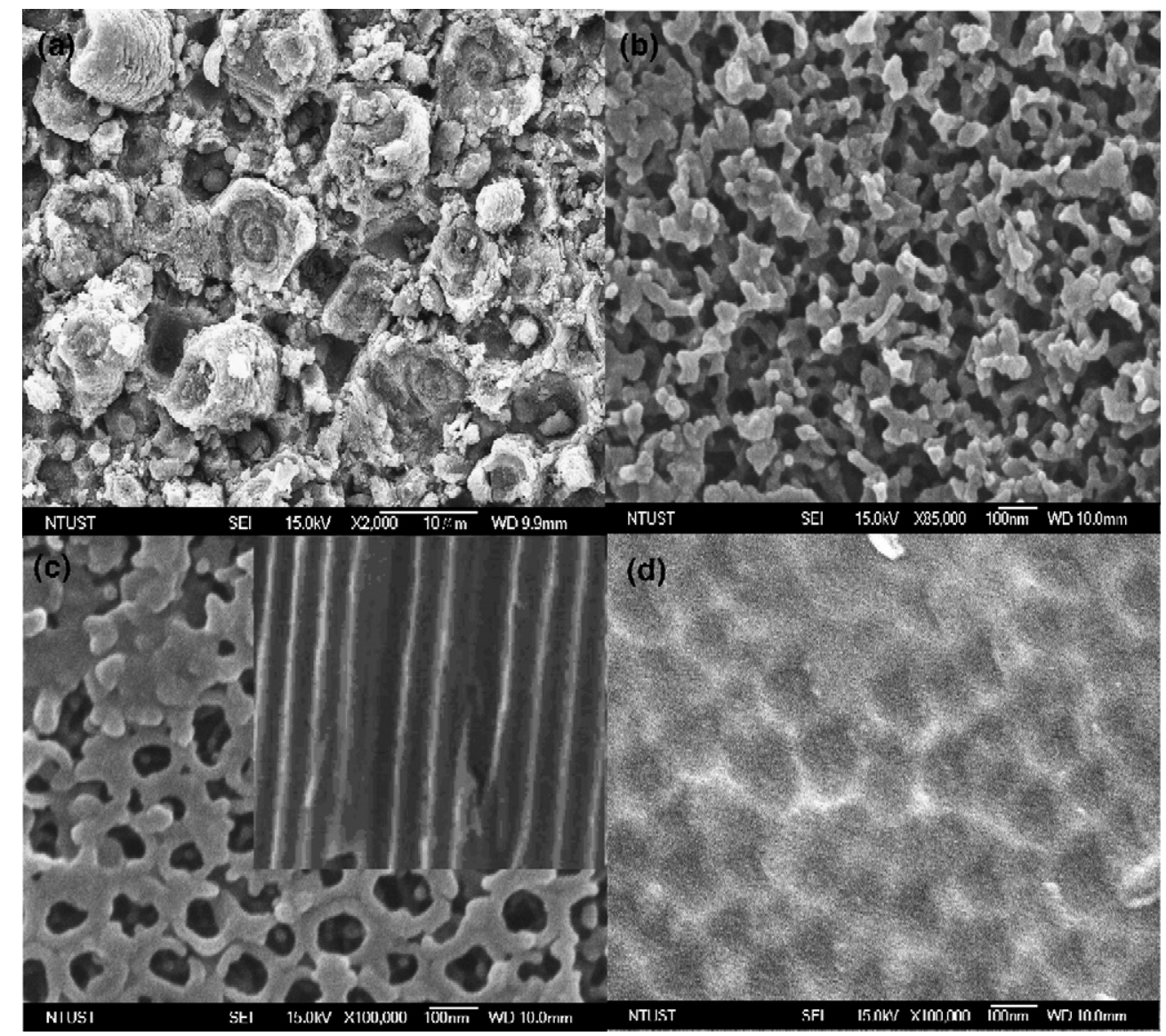

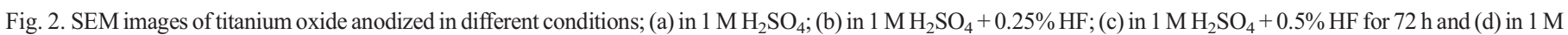
$\mathrm{H}_{2} \mathrm{SO}_{4}+0.5 \% \mathrm{HF}$ for $5 \mathrm{~h}$, at $40 \mathrm{~V}$. 


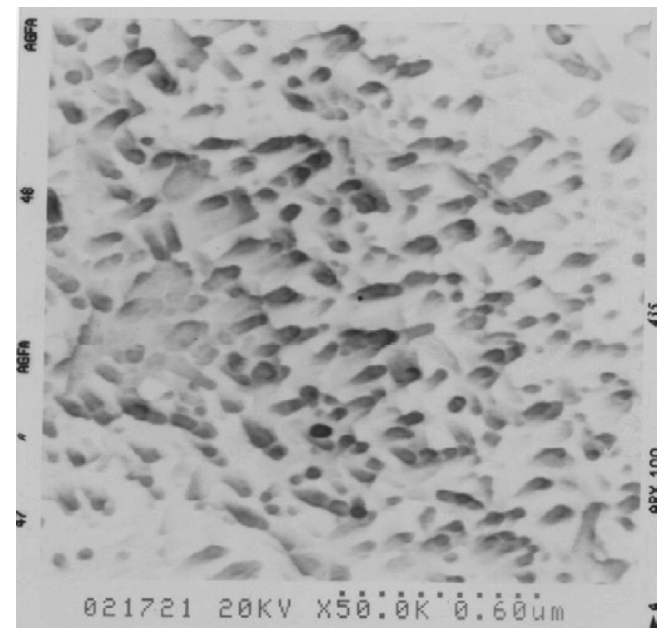

Fig. 3. SEM image of CNTs grown over ATO template before purification.

emerging. When the HF concentration is increased to $0.5 \%$ at $40 \mathrm{~V}$ for $72 \mathrm{~h}$, the intensity of the peaks for anatase and rutile phases is increased in a greater extent. Generally, XRD patterns show that when increasing the HF concentration in electrolyte under constant potential and anodization time, the anatase and rutile phases appear gradually. Further, it is observed that when increasing the $\mathrm{HF}$ concentration from 0 to $0.5 \%$, the intensity of the peaks of titanium on the XRD pattern is found to decrease significantly. The increase in peak intensity for $\mathrm{TiO}_{2}$ with a fall in intensity of $\mathrm{Ti}$ peaks with increasing $\mathrm{HF}$ concentration indicates that the formation of $\mathrm{TiO}_{2}$ with anatase and rutile phases increases with increasing HF concentration.

The morphology of the titanium surface anodized at different experimental conditions was analysed by SEM and the representative images are presented in Fig. 2a-d. It is observed from Fig. 2a, that the titanium surface anodized only in $1 \mathrm{M}$ $\mathrm{H}_{2} \mathrm{SO}_{4}$ for $72 \mathrm{~h}$ shows a rough surface without any regular pore formation indicating that anodization in $1 \mathrm{M} \mathrm{H}_{2} \mathrm{SO}_{4}$ at $40 \mathrm{~V}$ for $72 \mathrm{~h}$ does not lead to the formation of ordered pores, even though $\mathrm{TiO}_{2}$ is formed on the surface as revealed by the XRD analysis. When $0.25 \%$ of $\mathrm{HF}$ was introduced in the electrolyte and anodized for $72 \mathrm{~h}$, a sponge like structure (Fig. 2b) was observed without the appearance of uniform pores. Clear pores with a definite size are not seen on the surface. But structured pores with size of 70-80 nm were observed on the surface (Fig. 2c) of titanium metal anodized for $72 \mathrm{~h}$ in $1 \mathrm{M} \mathrm{H}_{2} \mathrm{SO}_{4}+0.5 \% \mathrm{HF}$ electrolyte solution at $40 \mathrm{~V}$. The cross-sectional view of ATO template presented as inset in Fig. 2c further confirming the formation of uniform channels, even though the outer surface of the template is not uniform due to anodization in acid medium for long time. The onset of pore formation is shown clearly in Fig. 2d, which is the SEM image of Ti surface anodized for $5 \mathrm{~h}$ in $1 \mathrm{M} \mathrm{H}_{2} \mathrm{SO}_{4}+0.5 \% \mathrm{HF}$ solution at $40 \mathrm{~V}$. It clearly shows the initiation of pore formation and the pore depth increases with increasing anodization time. Further, all the SEM images reflect that, several hours of continuous pore drilling at the inner interface and oxide dissolution are necessary to obtain uniform pores. The key processes for the formation of pores on Ti metal are (i) the oxide growth at the surface of the metal, (ii) metal ion $\left(\mathrm{Ti}^{4+}\right)$ migration from the metal at the metal/oxide interface under the applied electric field and (iii) field assisted dissolution of the oxide at the oxide/electrolyte interface [29,30]. When increasing the anodization time, the $\mathrm{Ti}$ ions are migrated to the solution, resulting in a significant loss of material and making the outer surface non-uniform. It may be due to the fact that titanium oxide can be etched at a high rate in the presence of HF solution. If the etching rate of the oxide in solution is comparable with that of the field-enhanced dissolution, the titanium oxide either in the wall or at the pore bottom will dissolve at a balanced rate resulting in a constant pore depth [24]. Because of the semiconducting nature of the $\mathrm{TiO}_{2}$, similar behaviors as those of alumina are not observed. Based on XRD and SEM analyses of anodized $\mathrm{TiO}_{2}$, the anodization for $72 \mathrm{~h}$ in $1 \mathrm{M} \mathrm{H}_{2} \mathrm{SO}_{4}+0.5 \%$ $\mathrm{HF}$ at a constant applied potential of $40 \mathrm{~V}$ was used for further experimental studies.

\subsection{SEM and TEM analyses of CNTS}

In order to study the morphology of as-grown CNTs before purification, SEM analysis was carried out and a typical image
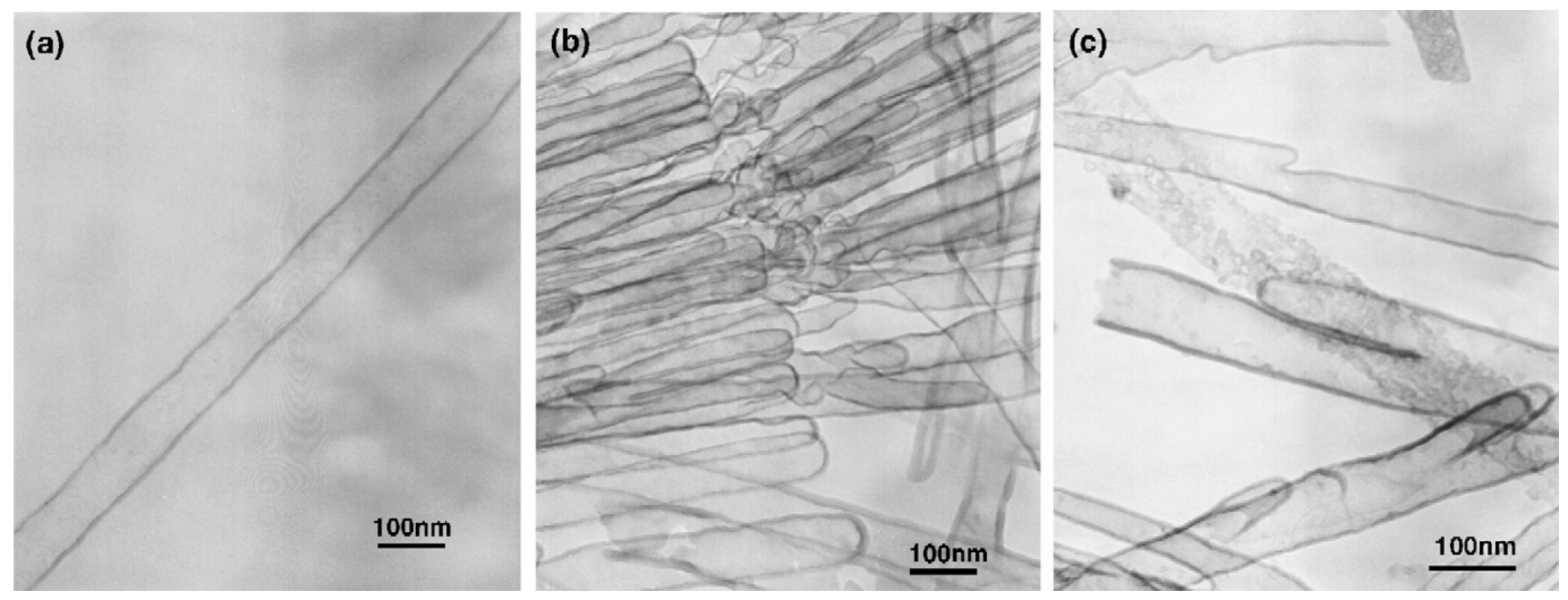

Fig. 4. TEM images of CNTs formed over ATO template. 

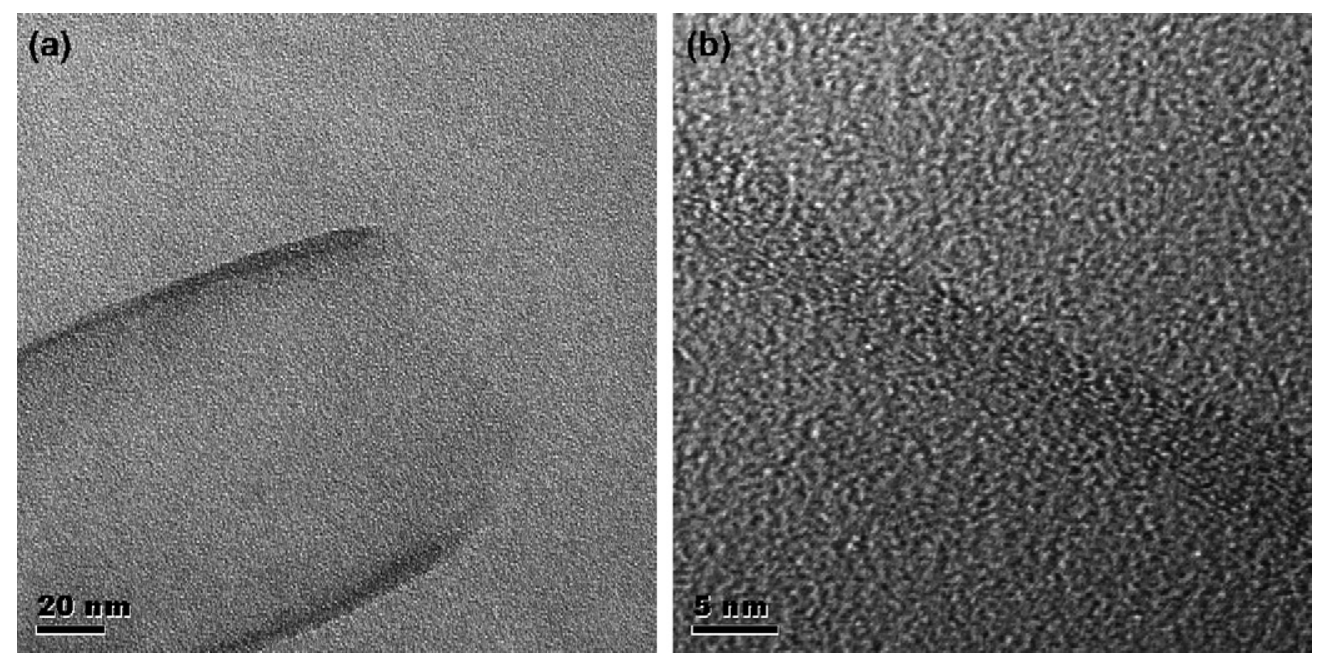

Fig. 5. HRTEM images of CNTs grown over ATO template.

is given in Fig. 3. It can be seen that the CNTs are grown from the pores of ATO and are confined inside the pores of the template. Most of the CNTs slightly overgrew out of the pores of the template. Also, the density of the grown CNTs is found to be higher, which reflects the pore density of ATO. The morphology of purified CNTs is analysed by TEM and the images are presented in Fig. 4. It is clearly seen that CNTs with diameter of $70-80 \mathrm{~nm}$ were observed. The thickness of the walls of the CNTs is found to be homogeneous. Also, the length of the formed CNTs is in the range of several micrometers with wide inner space. Further, most of the CNTs are found to be straight with very few of them having branches. In Fig. 4b, the closed end structure of the CNTs indicates that the CNTs are formed from the bottom of the pores (barrier layer). The open end structure is clearly noticed in Fig. 4c. The wall thickness is found to be very thin and uniform. Since, CNTs are used to copy the internal pore structure of the template, their morphological study gives a reliable approach to study the pore structure of ATO. The branched structure shown by the CNTs reflects the three-dimensional internal pore structure of the ATO template. Further, the walls of the CNTs are smooth with homogeneous thickness indicating the uniformity of the internal surface of pores. The domelike structure (closed end structure in Fig. 4b) of the bottom of CNTs is reflecting the structure of the so-called barrier layer, a thin oxidized layer separating the porous layer from the Ti metal substrate. This type of porous layer is commonly observed in porous alumina. When the CVD process is carried out at $650^{\circ} \mathrm{C}$, the decomposition of acetylene in the pores results in carbon deposition on the internal pore surface, which constitutes the tubes and copies the three-dimensional channel structure of the template. The formation of CNTs inside the ATO pores without any metal catalyst is a strong indication
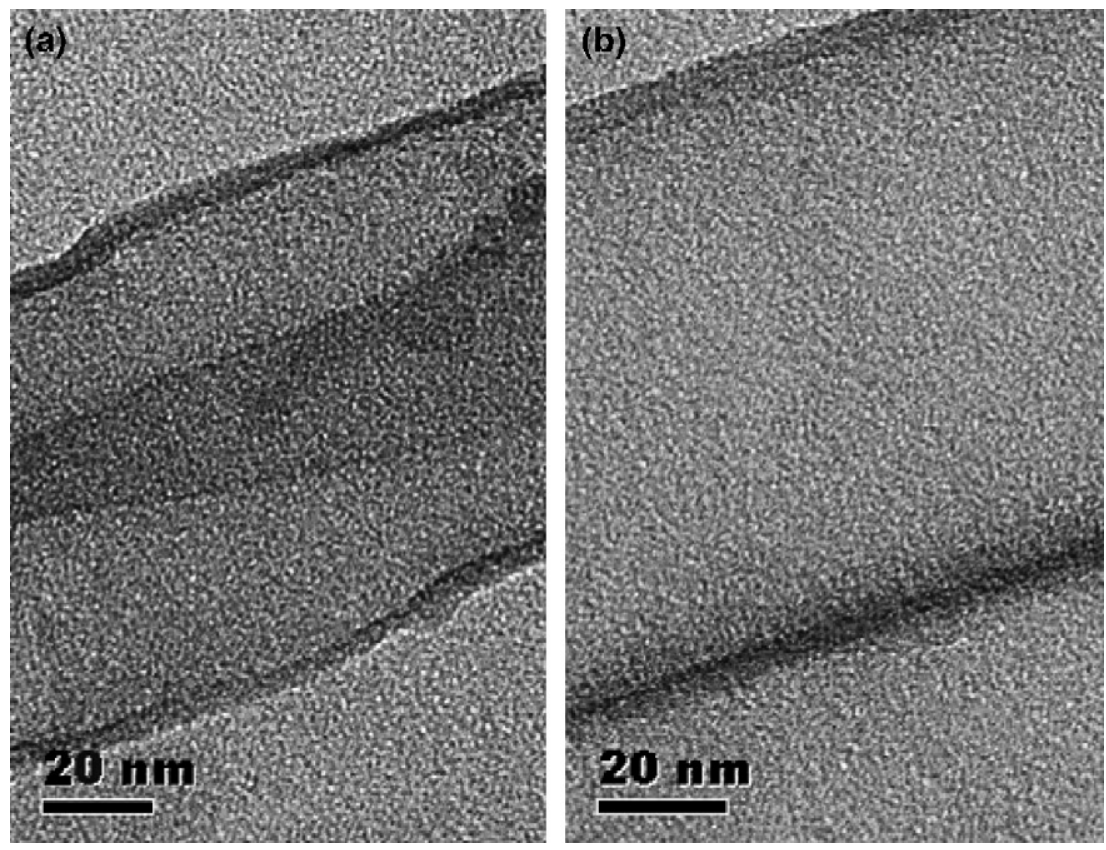

Fig. 6. HRTEM images showing the wall structure of CNTs grown with different deposition times; (a) $4 \mathrm{~h}$ and (b) $5 \mathrm{~h}$. 
(a)

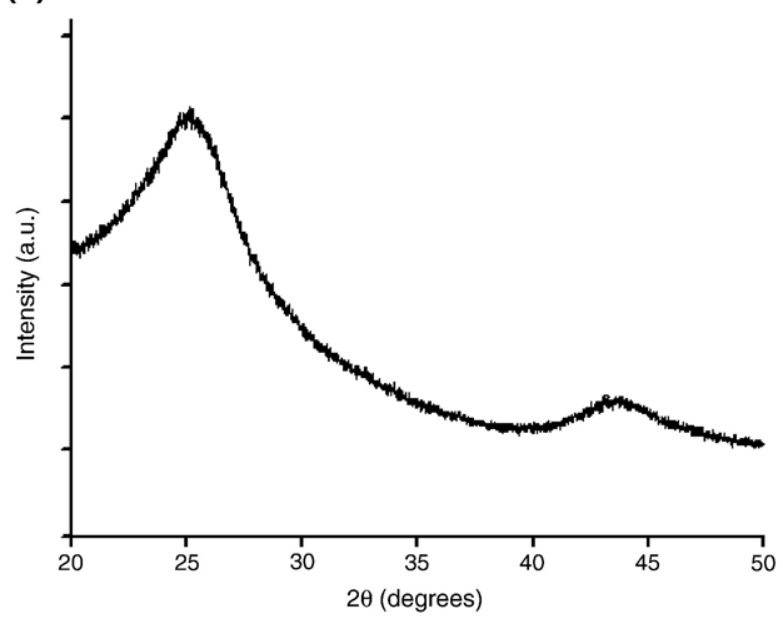

(b)

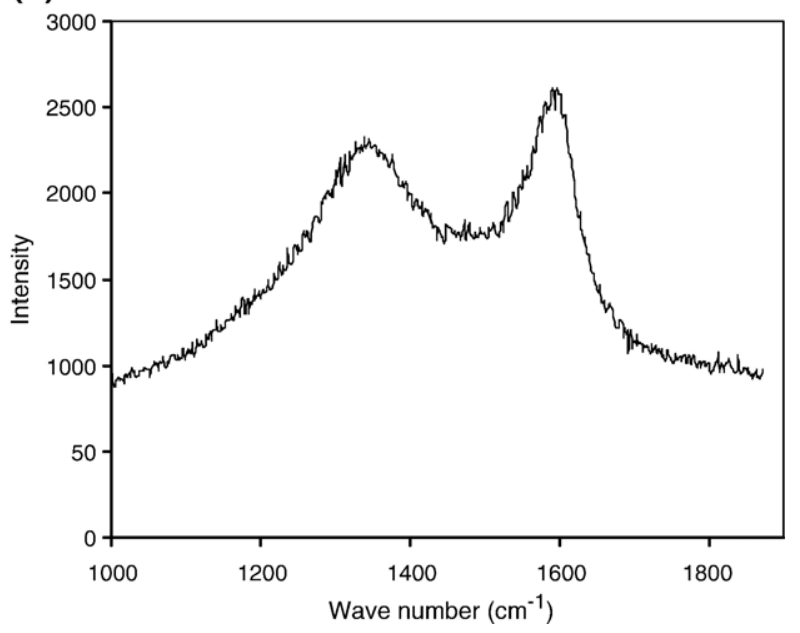

Fig. 7. XRD (a) and Raman spectrum (b) of CNTs grown over ATO template.

of the catalytic behavior of the internal pore surface of $\mathrm{TiO}_{2}$, in addition to template function. Hence, it is assumed that the Lewis acid sites of the $\mathrm{TiO}_{2}$ are responsible for their catalytic activity. Since, anatase and rutile forms of $\mathrm{TiO}_{2}$ are widely used as catalyst, catalyst support and photocatalyst in many chemical transformations, it is expected that the strength of Lewis acid sites of porous $\mathrm{TiO}_{2}$ is high enough to catalyse the decomposition of acetylene at $650{ }^{\circ} \mathrm{C}$. From the morphology of CNTs, it is assumed that the internal surface of pores is uniform and hence uniform catalytic activity is expected. Therefore, the chance for the decomposition of acetylene is the same at any point of the walls and hence the surface is uniformly covered by carbon deposits during the CVD process, leading to the formation of carbon nanotube-like structure.

\subsection{Tube wall structure from HRTEM analysis}

HRTEM measurements were also carried out to study the wall structure of the formed CNTs. Fig. 5a-b shows the HRTEM images of CNTs formed over the ATO template without Co metal catalyst. It is observed that the tubes have a relatively larger diameter and are found to be multi-walled. The wall thickness at the middle is in the range $2-3 \mathrm{~nm}$ (Fig. $5 \mathrm{~b}$ ) and the walls are found to have good crystallinity. The open end structure of the tips is also clearly noticed in Fig. 5a. Because of the amorphous background due to the specimen support film, the number of graphene layers is not clear. But, it can be guessed that the wall consists of around 5 graphene layers. It indicates that the CNTs are made of typical turbostratic graphite $[31,32]$. It is interesting to note that the internal diameter of the CNTs is around $70 \mathrm{~nm}$, which is different from that of CNTs grown over AAO [18], which is around $10 \mathrm{~nm}$. This is due to the fact that only a lesser number of graphene layers constitute the walls of CNTs, as shown by HRTEM. The CNTs are formed on the inner surface of the pores of the ATO template due to the catalytic decomposition of acetylene on Lewis acid sites of $\mathrm{TiO}_{2}$. The Lewis acid sites in the pores could act as a catalyst in forming few walls of the CNTs. But, after few walls are formed on the inner surface, the Lewis acid sites of ATO exist underneath the carbon nanotube walls. Due to this phenomenon, the Lewis acid sites of $\mathrm{TiO}_{2}$ can not act as a catalyst anymore and the catalytic decomposition of acetylene stops, typically resulting in a very thin wall as opposed to CNTs made by other methods. In order to confirm the same, CNTs were grown with
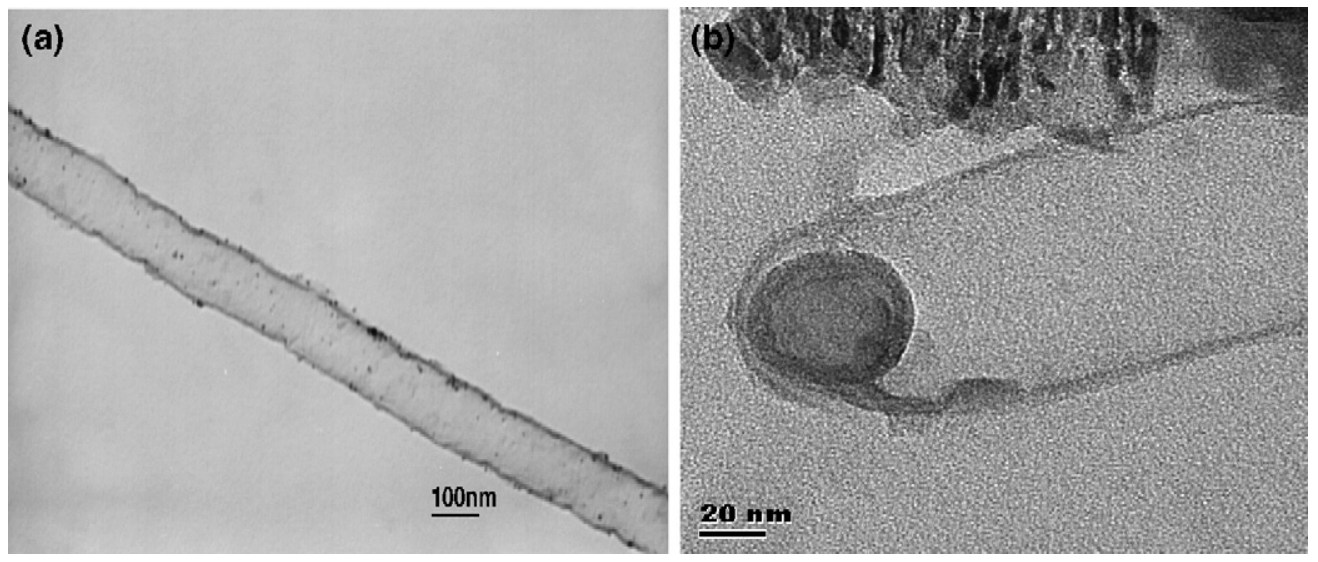

Fig. 8. TEM (a) and HRTEM (b) images of CNTs formed over anodized titanium oxide template with Co catalyst. 
deposition time of 4 and $5 \mathrm{~h}$ under similar experimental conditions and the corresponding HRTEM images are presented in Fig. 6a and b, respectively. The wall thickness of CNTs in both cases is around 2-3 nm. When comparing with that of CNTs with deposition time of $2 \mathrm{~h}$ (Fig. 5a), no significant increase in wall thickness is observed. It indicates that wall thickness of CNT is independent of carbon deposition time, which is opposite to the observation made by Kyotani et al. [18] over AAO template. The wall thickness is not increased by an increase in carbon deposition time indicating that the decomposition of acetylene, leading to formation of CNTs, is due to the Lewis acid sites of $\mathrm{TiO}_{2}$ and not due to the thermal decomposition.

\subsection{XRD and Raman spectroscopy of CNTs}

The structure and nature of the CNTs were analysed by X-ray diffraction and Raman spectroscopy and are presented in Fig. 7a and $b$, respectively. In XRD, two major peaks are observed, one is near $2 \theta=26^{\circ}$ corresponding to the 002 reflection, that related to the stacking order in polyaromatic solids. The other small asymmetric peak near $43.5^{\circ}$ is due to the 10 reflection of turbostratic carbon. The Raman spectrum shows two strong bands at 1347 and $1592 \mathrm{~cm}^{-1}$ confirming the multi-walled nature of the CNTs. These bands correspond to fundamental vibration modes of $\mathrm{D}_{6 \mathrm{~h}}^{4}$ of graphite [33]. The band at $1592 \mathrm{~cm}^{-1}$ ( $\mathrm{G}$ band) corresponds to the Raman allowed optical mode $\mathrm{E}_{2 \mathrm{~g}}$ of two-dimensional graphite, i.e. Raman active in-plane vibrational mode for an infinite or finite hexagonal network. The band at $1347 \mathrm{~cm}^{-1}$ (D band) is mainly derived from disordered carbon defects of the MWNTs, and is one of the in-plane vibrational modes. As compared to the $\mathrm{G}$ band at $1580 \mathrm{~cm}^{-1}$ for the graphitic carbons, the shift in $\mathrm{G}$ band towards a higher wavenumber $\left(1592 \mathrm{~cm}^{-1}\right)$ and the $I_{\mathrm{D}} / I_{\mathrm{G}}$ value $(0.88)$ suggest a turbostratic structure, consistent with HRTEM and XRD investigations.

For comparison purposes, CNTs were grown over Co electrodeposited ATO template following the same experimental conditions. The morphology of the formed CNTs after purification was analysed by TEM and HRTEM and the typical images are presented in Fig. 8a and b, respectively. When comparing the morphology as well as wall thickness of the CNTs formed over ATO with and without Co metal catalyst, no significant difference is observed. A metal particle inside the CNTs is clearly observed (Fig. 8b). But, still further detailed study is necessary to study the structural differences between the CNTs formed over the ATO template with and without Co catalyst.

\section{Conclusions}

Anodized titanium oxide with structured pores was obtained by an anodization process in $1 \mathrm{M} \mathrm{H}_{2} \mathrm{SO}_{4}+0.5 \% \mathrm{HF}$ at a constant potential of $40 \mathrm{~V}$ for $72 \mathrm{~h}$. Well ordered carbon nanotubes were formed over anodic titanium oxide template by CVD method using acetylene as carbon source. The CNTs are found to have good crystallinity with diameter of $70-80 \mathrm{~nm}$, similar to that of pores of the template. The formation of CNTs over ATO without metal catalyst indicates that the Lewis acid sites of $\mathrm{TiO}_{2}$ are having strong enough catalytic activity to decompose the acetylene, leading to the formation of CNTs. The TEM and HRTEM studies indicated that the CNTs are straight, hollow and multi-walled and the wall thickness is around 2-3 nm. The Lewis acid sites of $\mathrm{TiO}_{2}$ present in the internal surface of the pores play an important role in the catalytic decomposition of acetylene and the formation of CNTs. When increasing the carbon deposition time, the wall thickness of CNTs is not increased, indicating that the decomposition of acetylene is due to the Lewis acid sites of $\mathrm{TiO}_{2}$ and not due to thermal decomposition. Further, the XRD pattern confirms the turbostratic structure as well as good crystallinity of the CNTs. The Raman spectroscopy confirms the multi-walled nature of CNTs. There is no significant difference in morphology as well as wall thickness observed between the CNTs grown over ATO with and without cobalt catalyst. All the experimental results indicate that the ATO can be used as a template to grow well ordered high quality CNTs with a larger diameter. The selective formation of CNTs over porous titanium surface could be used for the fabrication of nanofunctional devices and the larger size of the CNTs favors higher metal dispersion as well as the accessibility of reactant molecules to metal particles inside the CNTs, when it is used as a catalyst support in various chemical reactions.

\section{Acknowledgement}

The authors gratefully acknowledge the financial support by the National Science Council of Taiwan through the grant NSC 93-2113-M-001-047.

\section{References}

[1] S. Fan, M.G. Chapline, N.R. Franklin, T.W. Tombler, A.M. Cassel, H. Dai, Science 283 (1999) 512.

[2] A.C. Dillon, K.M. Jones, T.A. Bekkedhal, C.H. Kiang, D.S. Bethune, M.J. Heben, Nature 386 (1997) 377.

[3] J.H. Hafner, C.L. Cheung, T.H. Oosterkamp, C.M. Lieber, J. Phys. Chem., B 105 (4) (2001) 743.

[4] L.P. Biro, S. Lazarescu, P. Lambin, P.A. Thiry, A. Fonseca, B. Nagy, J.B. Nagy, A.A. Lucas, Phys. Rev., B 56 (1997) 12490.

[5] S.J. Tans, A.R.M. Verschueren, C. Dekker, Nature 393 (1998) 49.

[6] R. Martel, T. Schmidt, H.R. Shea, T. Hertel, Ph. Avouris, Appl. Phys. Lett. 73 (1998) 2447.

[7] J.T. Hu, O.Y. Min, P.D. Yang, C.M. Lieber, Nature 399 (1999) 48.

[8] D.-J. Guo, H.-L. Li, J. Electroanal. Chem. 573 (2004) 197.

[9] S. Iijima, Nature 354 (1991) 56.

[10] B.J. Hinds, N. Chopra, T. Rantell, R. Andrews, V. Gavalas, L.G. Bachas, Science 303 (2004) 62.

[11] T. Ebbesen, P.M. Ajayan, Nature 358 (1992) 220.

[12] C. Journet, W.K. Maser, P. Bernier, A. Loiseau, M. Lamy de la Chapelle, S. Lefrant, P. Deniard, R. Leek, J.E. Fischer, Nature 388 (1997) 756.

[13] T. Guo, P. Nikolaev, A. Thess, D.T. Colbert, R.E. Smalley, Chem. Phys. Lett. 243 (1995) 49.

[14] M.J. Yacaman, M.M. Yoshida, L. Rendon, J.G. Santiesteban, Appl. Phys. Lett. 62 (1993) 202

[15] J. Li, C. Papadopoulos, J.M. Xu, Appl. Phys. Lett. 75 (1999) 367.

[16] J.S. Suh, J.S. Lee, Appl. Phys. Lett. 75 (1999) 2047.

[17] G. Che, B.B. Lakshmi, C.R. Martin, E.R. Fisher, Chem. Mater. 10 (1998) 260. 
[18] T. Kyotani, L.F. Tsai, A. Tomita, Chem. Mater. 8 (1996) 2109.

[19] L. Jing, M. Martin, H.L. Tom, Chem. Mater. 10 (1998) 1963.

[20] R. Asahi, T. Morikawa, T. Ohwaki, K. Aoki, Y. Taga, Science 293 (2001) 269.

[21] T. Albrektsson, P.I. Branemark, H.A. Hansson, J. Lindstrom, Acta Orthop. Scand. 52 (1981) 155.

[22] V. Zwilling, M. Aucouturier, E. Darque-Ceretti, Electrochim. Acta 45 (1999) 921.

[23] V. Zwilling, E. Darque-Ceretti, A. Boutry-Forveille, D. David, M.Y. Perrin, M. Aucouturier, Surf. Interface Anal. 27 (1999) 629.

[24] D. Gong, C.A. Grimes, O.K. Varghese, W. Hu, R.S. Singh, Z. Chen, E.C. Dickey, J. Mater. Res. 16 (12) (2001) 3331.

[25] R. Beranek, H. Hildebrand, P. Schmuki, Electrochem. Solid-State Lett. 6 (3) (2003) B12.
[26] J. Choi, R.B. Wehrspohn, J. Lee, U. Gosele, Electrochim. Acta 49 (2004) 2645.

[27] J.-H. Park, Y.-K. Lee, K.-M. Kim, K.-N. Kin, Surf. Coat. Technol. 195 (2-3) (2005) 252.

[28] B. Yang, M. Uchida, H.-M. Kim, X. Zhang, T. Kokubo, Biomaterials 25 (2004) 1003

[29] V.P. Parkhutik, V.I. Shershulsky, J. Phys. D: Appl. Phys. 25 (1992) 1258

[30] D.D. Macdonald, J. Electrochem. Soc. 140 (1993) L27.

[31] V. Ivanov, J.B. Nagy, P. Lambin, A.A. Lucas, X.B. Zhang, X.F. Zhang, D. Bernaerts, G. Vantendeloo, S. Amelinckx, J. Vanlanduyt, Chem. Phys. Lett. 223 (1994) 329.

[32] K. Hernadi, A. Fonseca, J.B. Nagy, D. Bernaerts, A.A. Lucay, Carbon 34 (1996) 1249.

[33] P.S. Eklund, J.M. Holden, R.A. Jishi, Carbon 33 (1995) 959. 